\section{DENTINE HYPERSENSITIVITY AND YOUR PATIENTS}

Colgate, a global leader in oral care and your partner in oral health, continues to share knowledge and experience through a series of complimentary educational webinars.

The next Colgate live webinar in the 2016 series, 'Dentine Hypersensitivity and your patients: 1 solution, 3 benefits', will be held on Tuesday 17 May 2016, 7.30-8.30 pm.

Join this exclusive event with Dr Peter Santarpia, Colgate's Manager of Technology and Dr Evaristo Delgado, Colgate's Associate Director of Technology to hear about:

- How to overcome the challenges of patients with dentine hypersensitivity

- How the breakthrough technology in Colgate Sensitive Pro-Relief toothpaste provides instant and long lasting relief from dentine hypersensitivity.
View and participate in this live webinar from the comfort of your home or practice by visiting www. colgateprofessional.co.uk/ webinars where pre-registration is available now.

The first 100 people to join the webinar will receive a Colgate ProClinical C250 Electric Toothbrush and all UK-based participants will be entered into a free prize draw for a Denlux DP2000 Digital Pulptester (upon completion of questionnaire at the end of the webinar; Tstcs apply).

Colgate Sensitive Pro-Relief toothpaste is the only sensitivity toothpaste to deliver instant and long lasting relief from dentine hypersensitivity (when applied to each affected tooth for one minute) along with the recommended fluoride level for cavity protection (1350-1500 ppm).

For further information visit www. colgateprofessional.co.uk.

\section{ALL THE LATEST PRODUCTS UNDER ONE ROOF}

Attend an evening of lectures, discussion and the opportunity to experience the GXDP-800 at the 'Progressing your Practice' live event series.

Chris Barrow, renowned consultant, trainer and coach to the UK dental profession, will be presenting his take on 'Marketing Dentistry in the current digital landscape' with what promises to be an engaging and entertaining lecture. You will also gain an insight into the ways 3D imaging can bring benefits to your practice with the 'Progressing your Practice with CBCT' lecture and take part in a hands-on demonstration of the latest 3D imaging system from Gendex, the GXDP-800.

KaVo, Kerr, Nobel Biocare, Orascoptic and Ormco will all be exhibiting at the events giving you access to the latest product innovations from these market leading companies all under one roof.

The events* are free to attend and you will receive up to three hours of verifiable CPD. What's more, you can choose the location that is most convenient for you:

London, 16 June 2016, 17.30-21.00

Birmingham, 27 June 2016, 17.30-21.00

Dublin, 8 September 2016, 18.00-20.00

Cardiff, 22 September 2016, 17.30-21.00.

Find out more and register now at go.kavo.com/uk/ progressingyourpractice

*Limited places available. You will receive an email confirming your place. KaVo Dental reserves the right to make changes or cancel the events as necessary. Chris Barrow will not appear at the event in Dublin.
ENSURE YOUR DEFIB MACHINE IS NOT JUST AN ORNAMENT

Endorsed by the GDC, the latest resuscitation guidelines require all clinical practices to have an AED (automated external defibrillator) immediately accessible by all staff, and the dental team should be capable using it.

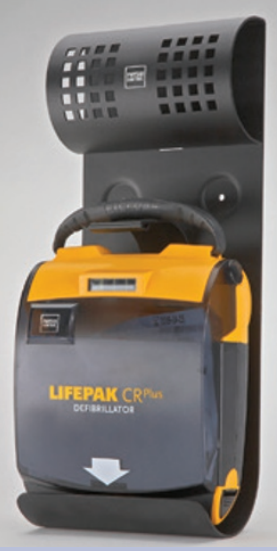

Defib Machines, supplier of fully serviced and maintained defibrillators, provide an all-inclusive package designed specifically for dental professionals: Dental Defibs.

For just £1 a day the Dental Defibs package will align your practice with both the GDC requirements and CQC scoring guidelines. The package includes:

- Full installation of Defib Machine(s)

- Onsite demonstration of use, using a non-live trainer unit

- Full maintenance service of machine, including regular onsite health checks and management of $\mathrm{pad} /$ battery replacement

- Replacement option for Defib Machine(s) after 18 months, if used in an emergency or displaying maintenance signals

- Resuscitation kit; signage; case; demo and training DVD

- Protective hard casing and adult live pads

- Certificate of full testing and results.

As an important part of the emergency medical equipment it is imperative that your device is cared for properly and not treated as an ornament. With Dental Defibs your device will receive regular health checks, in line with requirements, to make sure the device is in full working order for when it is needed most.

If you already have a Defib on site, but without a full replacement or servicing scheme, Defib Machines now provide a Buy-Back scheme. You will receive up to £250 for your outdated device, a brand new Defib Machine and our all-inclusive service and maintenance package.

Call 01274296842 for more information about Defib Machines and to arrange a free demonstration, or visit www.defibmachines.co.uk. 